\title{
Heartbeat: Anatomy versus physiology for diagnosis of coronary artery disease
}

The optimal approach to the initial diagnosis of coronary artery disease (CAD) in patients with chest pain symptoms is controversial. Historically, graded treadmill exercise stress testing for diagnosis of CAD was proposed in 1963 by Robert A. Bruce in Seattle because direct visualisation of the coronary arteries was expensive and risky at that time. Over the intervening five decades, a plethora of stress testing and imaging approaches have been developed, with persuasive advocates for each type of testing and guidelines stating that each is 'appropriate' but with little guidance for clinicians as to which test is best or should be performed first.

This controversy is addressed in this issue of Heart with an original research paper, a review article and two editorials. To investigate whether direct anatomic imaging of coronary anatomy improves outcomes in patients with suspected angina, The Scottish COmputed Tomography of the HEART (SCOT-HEART) Trial Investigators ${ }^{1}$ randomised 4146 patients to standard care with or without computed tomographic coronary angiography (CTCA). The previously reported primary findings ${ }^{2}$ showed that use of CTCA was associated with reduced rates of fatal and non-fatal myocardial infarction, better selection of patients for invasive angiography and more appropriate changes in therapy. However, despite objective improvement in clinical outcomes, patients did not feel better; the current study shows that CTCA was associated with slightly less improvement in symptoms and quality of life at 6 weeks and 6 months.

In an editorial, Cademartiri and

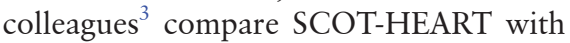
PROMISE (PROspective Multicenter Imaging Study for Evaluation of chest pain). In both studies, diagnosis of CAD was improved with CTCA. These authors argue that 'The possibility to stratify ${ }^{1}$ University of Washington School of Medicine, Seattle,
Washington, USA
${ }^{2}$ University Hospital and School of Medicine,
Universidade Federal de Minas Gerais, Belo Horizonte,
Brazil

Correspondence to Professor Catherine M Otto, Division of Cardiology, University of Washington, Seattle,WA 98195, USA; cmotto@uw.edu

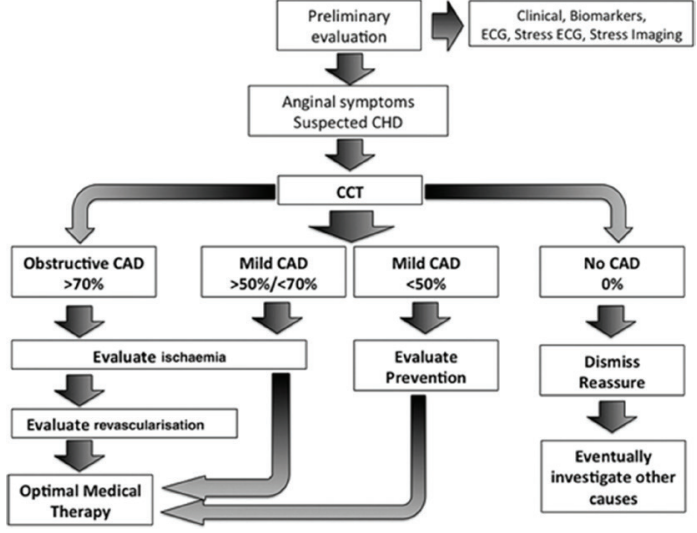

Figure 1 Proposed algorithm by Cademartiri et al. ${ }^{3}$ Evolving role of CCT in the workup of patients with suspected anginal chest pain. The figure shows a possible simplified algorithm which implements the potential role of CCT in patients with suspected anginal chest pain. While it is straightforward when significant obstructive disease is present ( $>70 \%$ stenosis), the management becomes more articulated when different degrees of obstruction are detected. CAD, coronary artery disease; CHD, coronary heart disease; CCT, cardiac CT.

patients according to their disease (type, severity, extension and so forth) and not according to general epidemiological risk classifications or according to the degree of ischaemia is an innovation,' (figure 1). Further, they point out that we have little data on outcomes and management of non-obstructive CAD; a diagnosis now easily made by CTCA. They conclude: 'Maybe, the true Copernican revolution at this stage should consist in realising that anatomy and function should no longer be seen as opponent, but should be used appropriately at different stages of the disease and also of the patient-physician relationship.'

The current National Institute for Health and Care Excellence (NICE) guidelines for diagnosis of stable chest pain have been revised recently to include CTCA as the key initial step in patient management as summarised in a review by Timmis and Roobottom. ${ }^{4}$ These guidelines now recommend an initial anatomic assessment of coronary anatomy by CTCA rather than physiologic stress testing for detection of myocardial ischemia, (figure 2). This article reviews the degree of uncertainty in clinical estimates of CAD probability (which are critical for a physiological testing approach), the sensitivity and specificity of physiological testing for diagnosis of obstructive CAD and the advantages of anatomical testing. They conclude 'The 2016 update to the NICE chest pain guideline marks a radical departure from the earlier diagnostic paradigm that will be welcomed by clinicians. No longer is there the recommendation to apply probability estimates of CAD to guide decisions about non-invasive diagnostic testing. Instead, the new recommendation is for CTCA in all patients with typical or atypical chest pain to determine whether there is obstructive CAD to account for the symptoms.'

In a provocative editorial, Cremer and Nissen ${ }^{5}$ provide a contrasting viewpoint. Their interpretation of the data from the SCOT-HEART and PROMISE trials in conjunction with pre-test probability estimates and stress testing leads them to conclude: 'Given that the NICE update would require a dramatic increase in cardiac CT scanners and accredited practitioners to report the scans, as well as expose many patients to unnecessary radiation and downstream testing, is the available evidence strong enough to support CTCA for all stable patients with typical or atypical angina? The answer is 


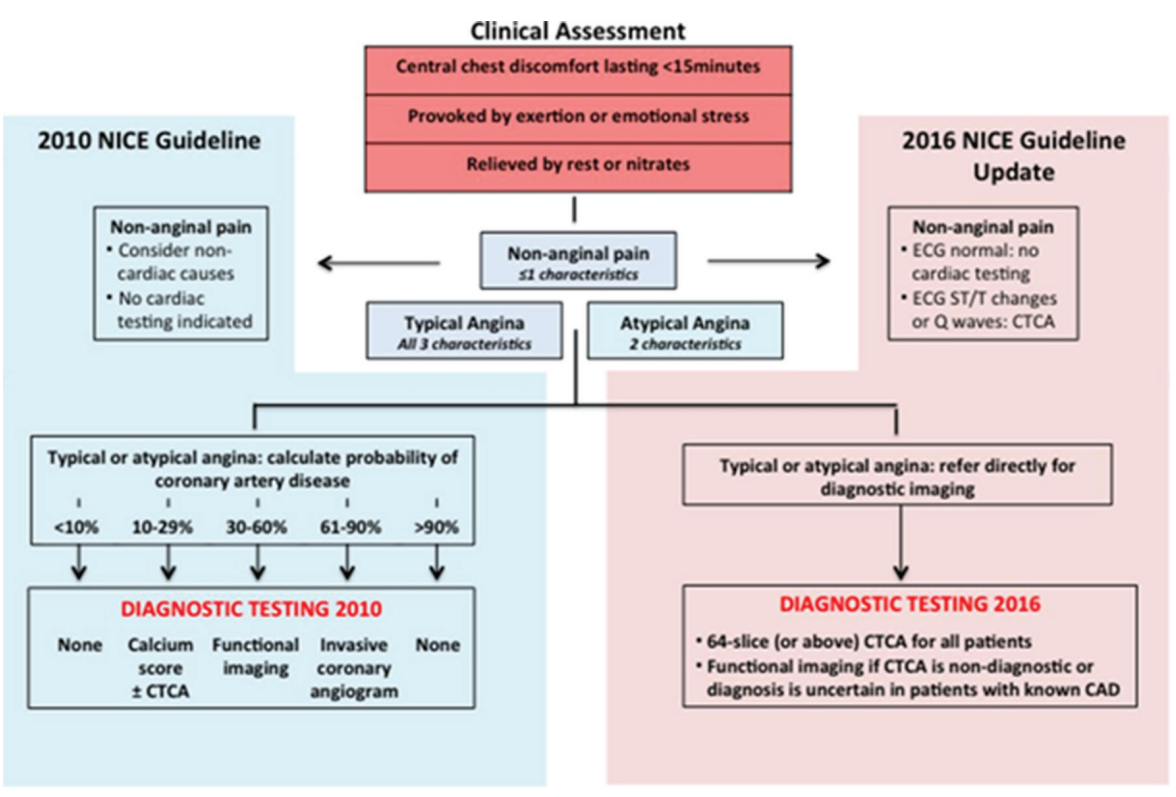

Figure 2 Diagnostic testing for people in whom stable angina cannot be excluded by clinical assessment alone: changes in NICE guideline recommendations 2010-2016. CAD, coronary artery disease; CTCA, CT coronary angiography; NICE, National Institute for Health and Care Excellence. ${ }^{4}$

a resounding 'No'. On the contrary, this update to the NICE guidelines should have focused on the low event rate achievable with optimal medical therapy and the inability of imaging to modify outcomes for many of these low-risk patients.'

Timmis and Roobottom rebut these arguments in a letter ${ }^{6}$ which details their response and concludes: 'NICE guidelines are evidence-based recommendations produced by an independent national body with experts in systematic review, data analysis and economic modelling. They gather all the credible scientific evidence and employ strategies that take into account the views of patients and national stakeholders to deliver cost effective guidelines for UK practice.' Even though Robert Bruce was my (CMO) mentor as a medical student aw

nd during cardiology training and even though I perform stress tests daily, I agree with Timmis and Roobottom that the evidence supports CTCA as the optimal first step in evaluation of patients with suspected angina. Again, from a historical point of view, physiological testing only became the standard because we were unable to routinely visualise coronary anatomy directly at that time; now we can. In addition, CTCA allows diagnosis of non-obstructive $\mathrm{CAD}$, a condition that has been inadequately characterised in terms of clinical outcomes and patient management. Certainly, physiological testing will continue to be important in many clinical situations to define the location and extent of myocardial ischemia. Halton's extension of Fisher's exact test. ${ }^{7}$
A second topic of interest in this issue of Heart is gender differences in the natural history, presentation, outcomes and healthcare practice in patients with atrial fibrillation (AF). Schnabel et $a l^{7}$ examined gender differences in symptoms, risk factors, therapies and 1 year incidence of adverse outcomes in a European contemporary cohort, the PREFER in AF registry. The sample consists of 6412 patients aged 18 years and older with physician-verified AF across seven Western European countries (France, Germany, Austria, Switzerland, Italy, Spain and the UK), enrolled from January 2012 to 2013 and followed for at least 1 year. Men and women differ in the time and mode of presentation; men with AF were on average younger than women $(70 \pm 11$ vs $74 \pm 10$ years) and women weremore symptomatic than men $(95.4 \%$ of women with at least one AF-related symptom vs $89.8 \%$ of men), (figure 3).

Anticoagulation usage was similar, but women wereless likely to receive invasive rhythm control therapy such as electrical cardioversion $(14.9 \%$ women vs $20.6 \%$ men) or ablation $(3.3 \%$ women vs $6.3 \%$ men). Women also had $40 \%$

Figure 3 Symptoms according to the European Heart Rhythm Association classification at baseline by gender. Percentages and $95 \% \mathrm{Cl}$ are provided. $\mathrm{p}$ Values are derived from Freeman-

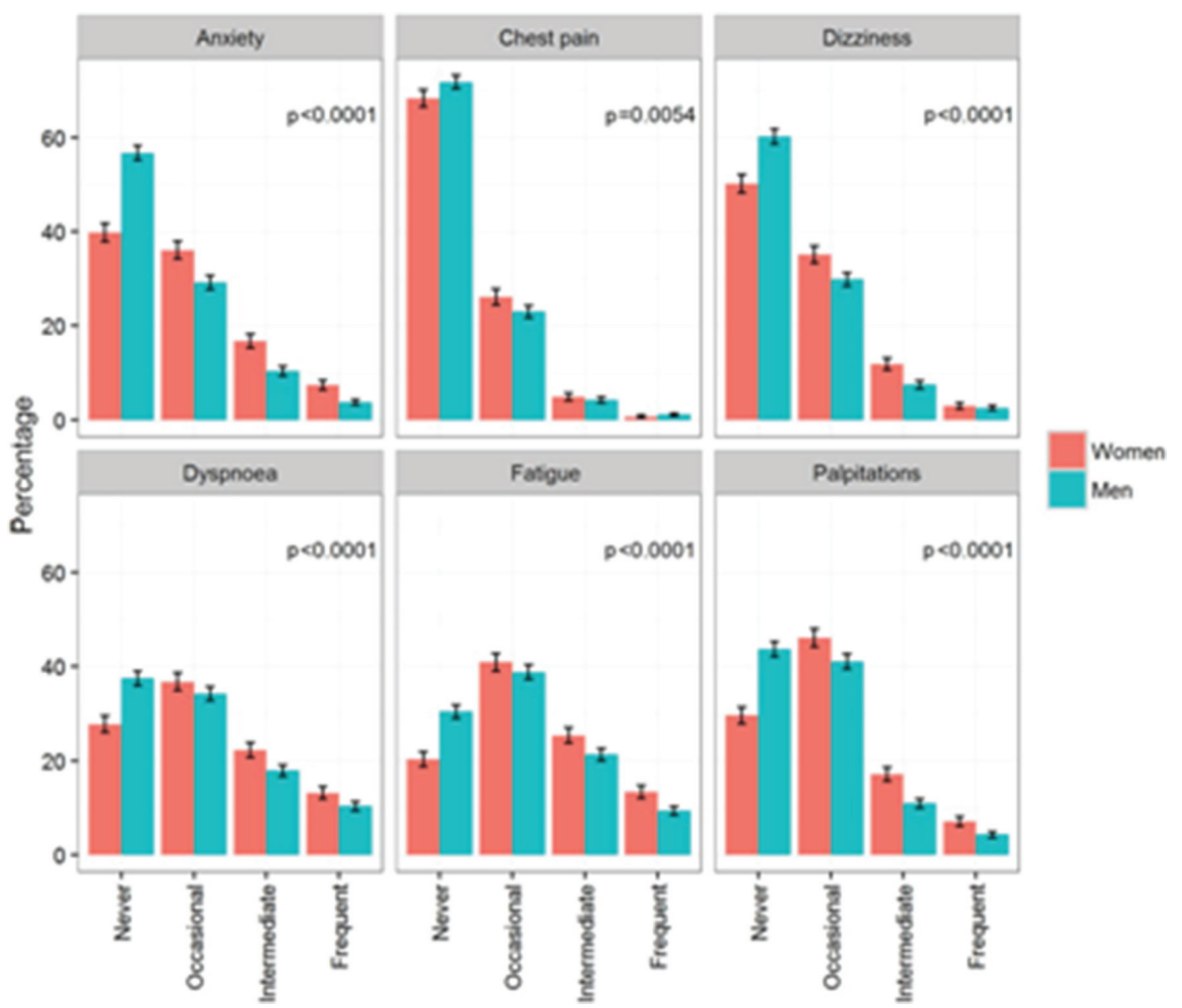


lower odds of developing acute coronary syndrome and 20\% lower odds of heart failure. The concluded that 'the observed gender disparities require detailed pathophysiological and clinical work-up, and may provide the opportunity to develop gender-specific preventive and therapeutic strategies for a disease that is reaching epidemic proportions worldwide'

In the companion editorial, Potpara and Blomstrom-Lundwqvist ${ }^{8}$ reviewed the main sex-related differences of atrial fibrillation under clinical and healthcare utilisation perspectives, pointing out that some differences on how men and women are being treated are due to to patient-related factors, including eligibility, the presence of contraindications to treatment and patient preferences.

The Education in Heart article in this issue provides a basic understanding of the epidemiology and management of spontaneous coronary artery dissection (SCAD). ${ }^{9}$ Most cases of SCAD occur in young to middle-aged women and may account for as much as $25 \%$ of acute coronary syndrome presentations in women $<50$ years of age, (figure 4$)$. The dissection flap is not always well visualised on angiography and a conservative approach to revascularisation is recommended, when possible.

\section{Competing interests None declared.}

Provenance and peer review Commissioned; internally peer reviewed.

(c) Article author(s) (or their employer(s) unless otherwise stated in the text of the article) 2017. All rights reserved. No commercial use is permitted unless otherwise expressly granted.

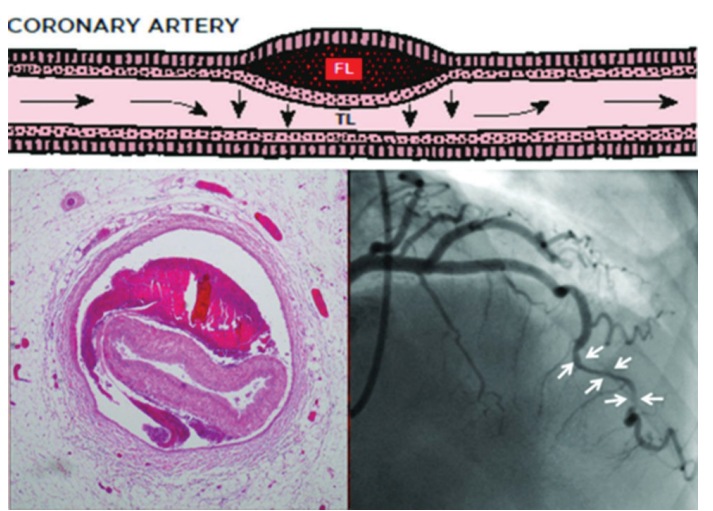

Figure 4 Pathophysiology of SCAD. A spontaneous haematoma forms in the outer media of a coronary artery forming an FL. This then compresses the artery from the outside restricting blood flow in the TL with typical histological and angiographic appearances. FL, false lumen; SCAD, spontaneous coronary artery dissection; TL, true lumen.

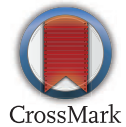

To cite Otto CM, Luiz Ribeiro A. Heart 2017:103:969-971.

Received 15 May 2017

Accepted 15 May 2017

Published Online First 1 July 2017

Heart 2017;103:969-971.

doi:10.1136/heartjnl-2017-311860

\section{REFERENCES}

1 Williams MC, Hunter A, Shah A, et al. Scottish COmputed Tomography of the HEART (SCOT-HEART)

Trial Investigators. Symptoms and quality of life in patients with suspected angina undergoing $\mathrm{CT}$ coronary angiography: a randomised controlled trial. Heart 2017;103:995-1001.

2 SCOT-HEART investigators. CT coronary angiography in patients with suspected angina due to coronary heart disease (SCOT-HEART): an open-label, parallelgroup, multicentre trial. Lancet 2015;385:2383-91.
3 Cademartiri F, Nistri S, Tarantini G, et al. Management of coronary artery disease with cardiac CT beyond gatekeeping. Heart 2017;103:975-6.

4 Timmis A, Roobottom CA. National Institute for Health and Care Excellence updates the stable chest pain guideline with radical changes to the diagnostic paradigm. Heart 2017;103:982-6.

5 Cremer PC, Nissen SE. The National Institute for Health and Care Excellence update for stable chest pain: poorly reasoned and risky for patients. Heart 2017;103:972-4.

6 Timmis A, Rootottom C. Re: the National Institute for Health and Care Excellence update for stable chest pain: poorly reasoned and risky for patients. Heart 2017; 103:1055.

7 Schnabel RB, Pecen L, Ojeda FM, et al. Gender differences in clinical presentation and 1-year outcomes in atrial fibrillation. Heart 2017;103:1024-30.

8 Potpara TS, Blomstrom-Lundqvist C Sex-related differences in atrial fibrillation: can we discern true disparities from biases? Heart 2017;103:979-81.

9 Al-Hussaini A, Adlam D. Spontaneous coronary artery dissection. Heart 2017;103:1043-51. 